\title{
Spin blockade and lifetime-enhanced transport in a few-electron Si/SiGe double quantum dot
}

\author{
NAKUL SHAJI ${ }^{1}$, C. B. SIMMONS ${ }^{1}$, MADHU THALAKULAM ${ }^{1}$, LEVENTE J. KLEIN ${ }^{1}$, HUA QIN ${ }^{1}$, H. LUO ${ }^{1}$, \\ D. E. SAVAGE ${ }^{1}$, M. G. LAGALLY1 ${ }^{1}$, A. J. RIMBERG ${ }^{2}$, R. JOYNT ${ }^{1}$, M. FRIESEN ${ }^{1}$, R. H. BLICK ${ }^{1}$, \\ S. N. COPPERSMITH ${ }^{1}$ AND M. A. ERIKSSON ${ }^{1 *}$ \\ ${ }^{1}$ University of Wisconsin-Madison, Madison, Wisconsin 53706, USA \\ ${ }^{2}$ Dartmouth College, Hanover, New Hampshire 03755, USA \\ *e-mail: maeriksson@wisc.edu
}

Spin blockade occurs when an electron is unable to access an energetically favourable path through a quantum dot owing to spin conservation, resulting in a blockade of the current through the $\operatorname{dot}^{1-6}$. Spin blockade is the basis of a number of recent advances in spintronics, including the measurement and the manipulation of individual electron spins ${ }^{7,8}$. We report measurements of the spin blockade regime in a silicon double quantum dot, revealing a complementary phenomenon: lifetimeenhanced transport. We argue that our observations arise because the decay times for electron spins in silicon are long, enabling the electron to maintain its spin throughout its transit across the quantum dot and access fast paths that exist in some spin channels but not in others. Such long spin lifetimes are important for applications such as quantum computation and, more generally, spintronics.

Semiconductor quantum dots or 'artificial atoms' provide highly tunable structures for trapping and manipulating individual electrons ${ }^{9-11}$. Such quantum dots are promising candidates as qubits for quantum computation ${ }^{12-14}$, owing in part to the long lifetimes and slow dephasing of electron spins in semiconductors ${ }^{7,15}$. Si quantum dots are predicted to have especially long lifetimes and slow dephasing, due to low spin-orbit interaction and low nuclear spin density ${ }^{16,17}$. In the past several years, much activity has focused on the development of quantum dots in Si/SiGe (refs 18-22) and recent advances in materials quality and fabrication techniques have enabled the observation of coherent spin phenomena in such quantum dots ${ }^{23}$.

Spin-to-charge conversion, in which spin states are detected through their effect on charge motion, enables measurement of individual electron spins in quantum dots ${ }^{15}$. Spin blockade is the canonical example of spin-to-charge conversion in transport, where charge current is blocked in a double quantum dot by a metastable spin state. The blockade occurs when one electron is confined in the left dot and a further electron enters the right dot forming a spin triplet state $\mathrm{T}(1,1)$ (Fig. 1a). Exiting the dot requires reaching the triplet $\mathrm{T}(2,0)$, with both electrons in the left dot, a state that is higher in energy. The electron is thus trapped in the right dot, unless relaxation from $\mathrm{T}(1,1)$ to $\mathrm{S}(1,1)$ occurs, opening a downhill channel through $S(2,0)$. As we show below, this aspect of spin blockade in $\mathrm{Si}$ is virtually identical to that previously observed in other systems ${ }^{1-3}$.

The unexpected effect presented here is lifetime-enhanced transport (LET). The energy level diagram for LET is the same as for spin blockade, except that current flows in the opposite direction (Fig. 1b). Flow through the triplet channel is now energetically downhill, whereas flow through the singlet channel is very slow, because it requires either an uphill transition or tunnelling directly from the left dot to the right lead. Transport current will be observable only if electrons flow almost exclusively through the triplet channel, requiring even slower triplet-singlet relaxation rates than those needed to observe spin blockade.

The tunable quantum dot used in these experiments was formed in a Si/SiGe heterostructure. The gate structure (Fig. 2a) has the shape often associated with a single quantum dot, and the corresponding Coulomb diamonds are shown in Fig. 2b. By tuning the gate voltages, the single dot was split into two tunnel-coupled quantum dots. Such transformations of a lateral single quantum dot into multiple quantum dots have been demonstrated in similar systems $s^{24,25}$. Here, by changing voltages on gates $\mathrm{G}$ and CS, and keeping those on $\mathrm{B}_{\mathrm{L}}, \mathrm{T}$ and $\mathrm{B}_{\mathrm{R}}$ fixed, the electron occupations are tuned while keeping the tunnel barriers constant (Fig. 2d). The left dot is coupled more strongly to gate $\mathrm{G}$, and the right dot is coupled more strongly to gate CS. The electron occupancies indicated in the figure correspond to an equivalent charge configuration with a single unpaired spin in the $(1,0)$ state.

The region of interest here is indicated by the blue dashed circle in Fig. 2d. The lower of these 'triple points' corresponds to degeneracy between the $(1,0),(1,1)$ and $(2,0)$ charge states $^{26,27}$. In this regime, an electron with a spin $|\uparrow\rangle$ or $|\downarrow\rangle$ is confined in the left dot, and the incoming electron can form either a spin singlet $\mathrm{S}:(|\uparrow \downarrow\rangle-|\downarrow \uparrow\rangle) / \sqrt{2}$ or any of the spin triplets $\mathrm{T}_{+}:|\uparrow \uparrow\rangle, \mathrm{T}_{-}:|\downarrow \downarrow\rangle$ or $\mathrm{T}_{0}:(|\uparrow \downarrow\rangle+|\downarrow \uparrow\rangle) / \sqrt{2}$, which are degenerate at zero magnetic field. The singlet-triplet energy splitting is larger for two electrons occupying the same dot $(2,0)$, than when they are in different dots $(1,1)$, resulting in the energy level schematic diagrams shown in Fig. 1.

Spin blockade arises because spin is conserved during tunnelling, preventing the direct transition from the triplet $\mathrm{T}(1,1)$ 

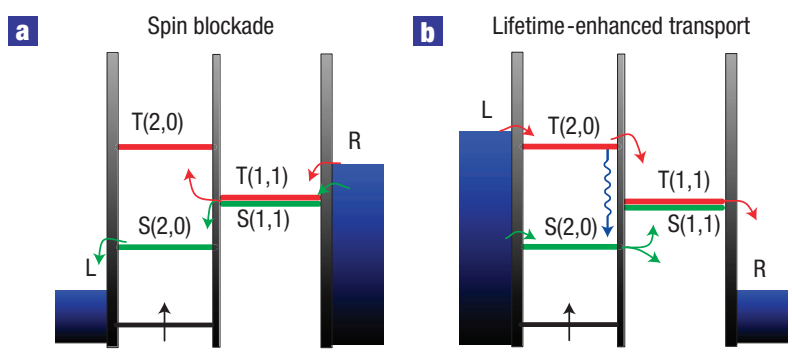

Figure 1 Pauli spin blockade regime in a double quantum dot. a, Energy level schematic diagram of a double quantum dot with one electron confined in the left dot (black). The applied bias causes electron flow from the right lead (R) to the left (L). Incoming electrons can form either a spin singlet $\mathrm{S}(1,1)$ (green) or a spin triplet $\mathrm{T}(1,1)$ (red). Electrons forming a singlet have an accessible fast channel through $S(2,0)$ to the left lead. In contrast, electrons entering the triplet $T(1,1)$ cannot exit through $\mathrm{T}(2,0)$, resulting in metastable occupation of the $\mathrm{T}(1,1)$ state, and causing spin blockade of the current. $\mathbf{b}$, The identical energy level configuration as in $\mathbf{a}$, but with the direction of current flow reversed. Electrons entering $S(2,0)$ have no fast path through the system. If $S(2,0)$ loads more rapidly than it unloads, current will be blockaded. In contrast, electrons entering $\mathrm{T}(2,0)$ have an accessible fast channel through $\mathrm{T}(1,1)$, provided spin relaxation to $\mathrm{S}(2,0)$ (blue wavy arrow) does not occur. Electron transport through the triplets, supported by a long spin lifetime, is denoted LET.

to the singlet $S(2,0)$. We observe this blockade as shown in Fig. 3a-c. These measurements are taken at finite bias, where the triple points expand into bias triangles ${ }^{28}$. When $T(1,1)$ is loaded and no relaxation occurs from $\mathrm{T}(1,1)$ to $S(1,1)$, spin blockade is observed (as marked by the orange triangle) and the bias triangle is truncated as shown schematically in Fig. 3b. The observed current in the blockaded region is limited by the noise floor in the measurement ( $7 \mathrm{fA}$ r.m.s.). Spin blockade is fully lifted when the $\mathrm{T}(2,0)$ state is brought below the $\mathrm{T}(1,1)$ state (blue star).

Now consider the same energy level configuration, but with opposite bias across the dot (Fig. 1b). In previous work, this configuration has been shown to be blockaded ${ }^{2,8}$. In contrast, here in Si we observe a strong 'tail' of current in this configuration, corresponding to the extra parallelograms (green outline) in Fig. 3d,e. As shown in detail below, the condition for observing this tail is that the metastable $S(2,0)$ state must be loaded more slowly than it empties. The relaxation rate from the $\mathrm{T}(2,0)$ state into $\mathrm{S}(2,0)$ sets a lower bound for this loading rate. Because the measured current at the point labelled $(+)$ is significant only when the spin lifetime of $\mathrm{T}(2,0)$ is long, we denote this tail of current the triplet tail and the effect LET.

The dimensions of the triplet tail in the charge stability diagram (Fig. 3d,e) provide a measurement of the energy difference between the $(2,0)$ triplet and singlet states $\left(E_{\mathrm{ST}}=E_{\mathrm{T}}-E_{\mathrm{S}}\right)$. Both the length of the tail and the distance between the tail and the edge of the bias triangle correspond to $E_{\mathrm{ST}}$ (Fig. 3e). This $(2,0)$ singlet-triplet energy gap as extracted from the data is $240 \pm 30 \mu \mathrm{eV}$.

A simple rate model gives insight into when LET occurs. The rates in the model correspond to transitions between the states shown in Fig. 1b, and the corresponding lifetimes are the inverses of the rates. By calculating the expected amount of time required for an electron to pass through the system, we obtain a quantity proportional to the measured current $I$ (see Supplementary Information for the complete analysis). The slow rates are of interest here: the relaxation rate $\Gamma_{\mathrm{TS}}$ from the triplet $\mathrm{T}(2,0)$ to the singlet $\mathrm{S}(2,0)$, the loading rate $\Gamma_{\mathrm{LS}}$ of the singlet $\mathrm{S}(2,0)$ from the lead and the unloading rate $\Gamma_{\mathrm{S}}$ of the singlet a

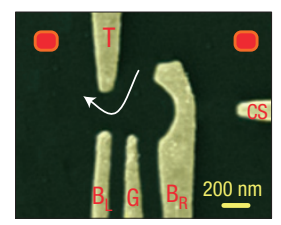

b
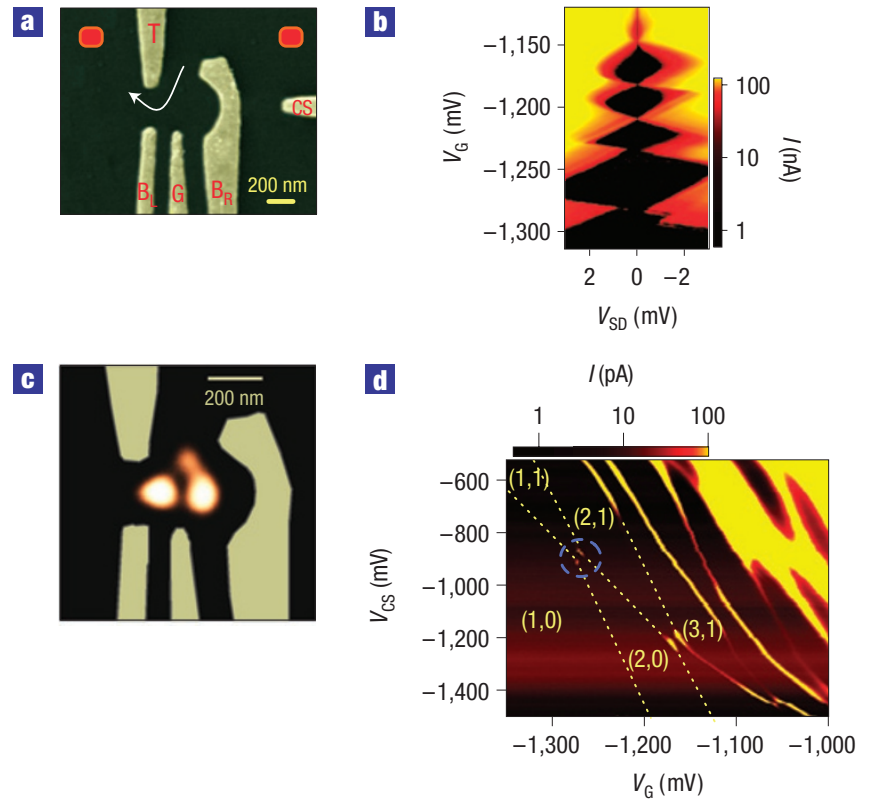

Figure 2 Formation of a double quantum dot. a, False-colour micrograph of a device similar to the one used in the experiments. A 2DEG was formed in a $12 \mathrm{~nm}$ strained silicon quantum well with a sheet carrier density of $4 \times 10^{11} \mathrm{~cm}^{-2}$ and a mobility of $40,000 \mathrm{~cm}^{2} \mathrm{~V}^{-1} \mathrm{~s}^{-1}$. Ohmic contacts (indicated schematically by red squares) were formed by annealing an alloy of $\mathrm{Au}: \mathrm{Sb}(1 \%)$ at $550^{\circ} \mathrm{C}$. Metal gates used to form the quantum dots were realized by depositing palladium on the sample surface and are labelled on the micrograph. The white arrow indicates the direction of electron flow when $V_{S D}>0$. b. Magnitude of the measured current as a function of source-drain voltage $V_{S D}$ and the voltage on gate $G$. The black regions indicate Coulomb blockade where the number of electrons in the dot is fixed. Outside this blockade, single-electron tunnelling through the dot occurs. c, A numerical simulation of the charge density for the gates as shown and for gate voltages corresponding to the double quantum dot data (d). Two electrons prefer to occupy opposite sides of the open region between the gates. $\mathbf{d}$, The single quantum dot was deformed into two tunnel-coupled dots in series by using a combination of negative voltages on gates $T, B_{L}$ and $B_{R}$. The magnitude of the measured current through the double quantum dot is plotted as a function of the voltages on gates $G$ and $C S$, with $V_{S D}=0.1 \mathrm{mV}$. The dot coupled more to gate $\mathrm{G}$ (CS) is the left (right) dot. As described in the text, the notation $(m, n)$ represents the effective left and right dot electron occupancy, and the triple point studied in detail here appears inside the blue circle.

$\mathrm{S}(2,0)$. To focus on these rates and to develop intuition, we assume that all other rates are equal to a single rate, $\Gamma_{\text {fast }}$, an assumption that does not change the qualitative understanding. The resulting proportionality for the current is

$$
I \propto \frac{\Gamma_{\text {fast }}}{3+\left(\Gamma_{\mathrm{TS}}+\Gamma_{\mathrm{LS}}\right) / \Gamma_{\mathrm{S}}} .
$$

As this proportionality shows, the triplet tail is observed if and only if the sum of the triplet-singlet relaxation rate $\Gamma_{\mathrm{TS}}$ and the loading rate from the lead $\Gamma_{\mathrm{LS}}$ is not large compared with the escape rate $\Gamma_{\mathrm{S}}$. If the triplet-singlet relaxation rate is much faster than the escape rate, then the tail regime will be blockaded by electrons trapped in the $S(2,0)$ state. In our experiments, essentially no reduction in current $(\sim 5 \%)$ is observed moving from the bias triangle into the tail (from the blue diamond towards the teal cross in Fig. 3d). Thus, electrons are rarely trapped in $S(2,0)$, indicating 

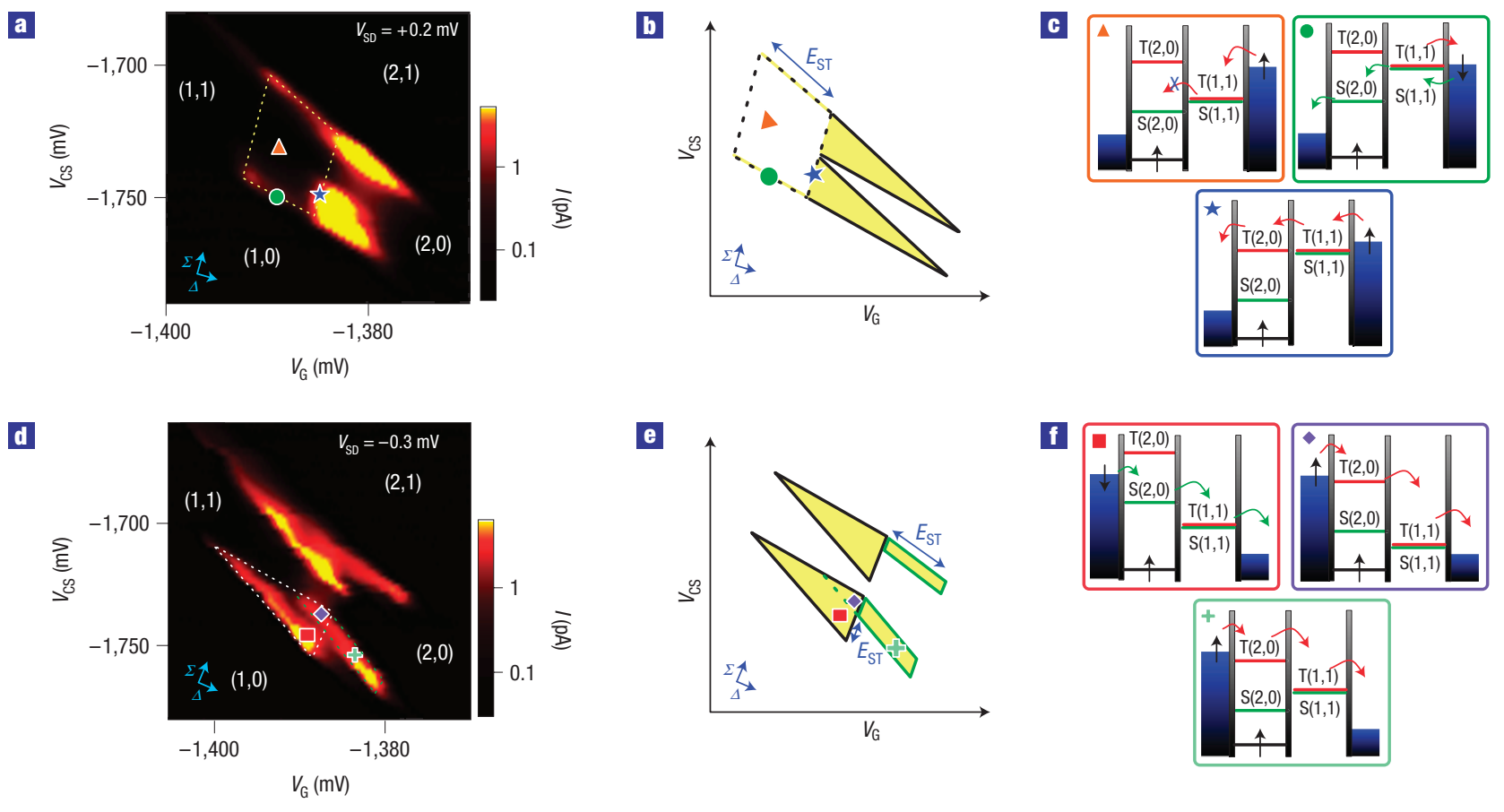

Figure 3 Spin blockade and LET. a, Positive bias $\left(V_{S D}=0.2 \mathrm{mV}\right)$ triangles representing the $(1,0)-(1,1)-(2,0)-(2,1)$ charge transition. Current through the device is blocked owing to Pauli spin blockade (as marked by the orange triangle) in the region outlined by yellow dashed lines. The blockade is lifted when the triplet state becomes accessible (blue star). The lower triangle is referred to as the 'electron triangle' and the upper triangle is called the 'hole triangle'. The blue arrows represent the energy axis $\Sigma$ where the energy levels of both dots are changed together, and the detuning axis $\Delta$ where dot levels are moved in opposite directions. Note that the edges of the spin blockade regime (green circle) show measurable current flow due spin exchange with the leads ${ }^{2}$. $\mathbf{b}$, Schematic representation of the positive bias triangles. Dashed lines mark the blockaded region, whereas filled yellow regions indicate no blockade. The energy gap between the spin singlet and triplet $\left(E_{S T}\right)$ is indicated along the edge of the triangles. c, Transport details and energy level schematic diagrams for the electron triangle corresponding to the points denoted by the green circle, orange triangle and blue star in a and $\mathbf{b}$. The sigma directions in $\mathbf{a}$ and $\mathbf{b}$ correspond to moving the levels on the left and right up and down together, and the delta directions correspond to moving the levels on the left and the right in opposite directions. The same pattern holds for parts $\mathbf{d}-\mathbf{f}$. $\mathbf{d}$, Negative bias $\left(V_{\mathrm{SD}}=-0.3 \mathrm{mV}\right)$ triangles through the same charge transition. Current flow through full electron and hole triangles is observed (white dashed lines are shown on the electron triangle). In addition, strong 'tails' of current (green dashed parallelograms) are observed at the base of the triangles. These extensions arise due to LET, as discussed in the text. e, Schematic representation of the negative bias triangles. Regions outlined by the solid back lines correspond to conventional transport, whereas those outlined by solid green lines (the tails) correspond to LET. Lengths corresponding to $E_{\mathrm{ST}}$ are labelled. f, Transport details and energy level schematic diagrams for the electron triangle corresponding to the points denoted by the red square, purple diamond and teal cross in $\mathbf{d}$ and $\mathbf{e}$.

that the triplet-singlet relaxation rate $\Gamma_{\mathrm{TS}}$ and the loading rate from the left lead $\Gamma_{\mathrm{LS}}$ are both much less than $\Gamma_{\mathrm{S}}$, itself a slow rate. A similar calculation with the opposite bias shows that the condition for spin blockade is that the triplet-singlet relaxation rate in the $(1,1)$ configuration is much slower than the fast rates, a far less stringent condition.

To investigate the rapid tunnelling between dots 1 and 2, and to understand the device physics, we have modelled the device numerically, as shown in Fig. 2c. Established methods are used to treat the various charge regions self-consistently, including trapped surface charge, ionized dopants, the two-dimensional electron gas (2DEG) and the device ${ }^{29}$. The dopants are treated in the jellium approximation, whereas the inhomogeneous depletion of the 2DEG is treated semiclassically in a 2D Thomas-Fermi approximation. For the gated region, a 2D Hartree-Fock basis of single-electron orbitals is obtained from the effective mass envelope equation, and a two-electron singlet wavefunction is constructed using the configuration interaction method, similar to ref. 14. The results show that the bottom of the quantum dot confinement potential is nearly flat, with an oblong shape about $200 \mathrm{~nm}$ across. General arguments suggest that the electron-electron interactions should dominate the kinetic energy in silicon for electrons separated by over $100 \mathrm{~nm}$, causing two electrons to form a double dot. The modelling results, shown in Fig. $2 c$, confirm that these general arguments give the correct intuition. As is clear from the figure, the effective tunnel barrier between the two dots is low, consistent with LET. We note that quantum dot splitting has been observed elsewhere, where it was attributed to deformation by a gate potential ${ }^{24}$ or a local impurity ${ }^{25}$. Although inhomogeneous confinement may also be present in our device, it is not needed to explain the double dot.

LET should be observable in many materials systems, provided the appropriate ratio of rates can be obtained. Indeed, slow triplet-singlet relaxation and the preferential loading of triplets versus singlets have both been observed in GaAs quantum dots, in pulsed-gate experiments ${ }^{30}$. By analysing the current versus voltage data in our bias triangles (see the Supplementary Information), we find that in the tail regime, triplet loading occurs at a rate at least 1,000 times greater than singlet loading. This ratio is 50 times greater than in previous observations of spin-dependent tunnelling ${ }^{30}$, which may lead to corresponding enhancements in spin readout. The singlet loading is suppressed because its tunnel barrier to the external lead is larger than that of the triplet state ${ }^{31}$; the relatively large effective mass of $\mathrm{Si}$ enhances this suppression. 

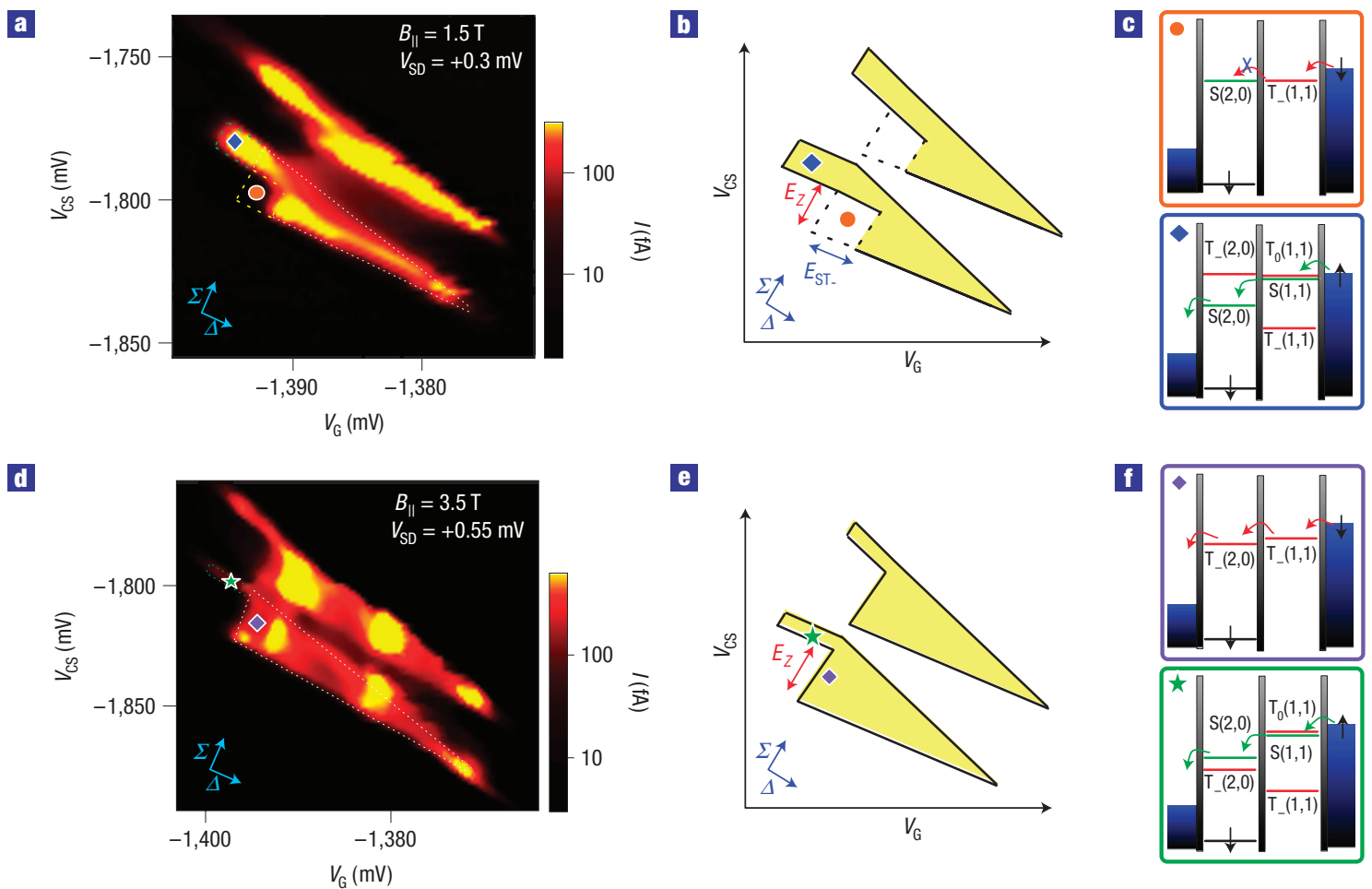

Figure 4 Zeeman splitting, spin blockade and LET. a, Magnitude of the measured current with an inplane magnetic field of $1.5 \mathrm{~T}$ applied such that $E_{Z}$ is less than $E_{\mathrm{ST}}$. Spin blockade is reduced to a smaller region indicated by dashed yellow lines. Note that there is no conduction along the edges because the $S(1,1)$ state is no longer energetically accessible when the $(1,1)$ ground state $T_{-}(1,1)$ aligns with the lead, preventing spin exchange. In the region labelled with a blue diamond, a tail appears corresponding to transport through the excited spin singlet state. $\mathbf{b}$. Schematic illustration of the bias triangles for small inplane magnetic field. The energies $E_{Z}$ and $E_{\mathrm{ST}-}=E_{\mathrm{ST}}-E_{Z}$ can be extracted from the graph as indicated by red and blue arrows. The angles in this schematic diagram are exaggerated relative to a. c, Energy level schematic diagrams at the points labelled by the orange circle and blue diamond in $\mathbf{a}$ and $\mathbf{b}$. d. Magnitude of the measured current with an inplane magnetic field of $3.5 \mathrm{~T}$ applied such that $E_{Z}$ is larger than $E_{\mathrm{ST}}$. No blockade is observed inside the triangles. Excited states are observed inside the triangles as bright lines parallel to the triangle bases. The tail regions correspond to LET through excited spin singlet states. e, Schematic illustration of the bias triangles for large inplane magnetic field. The energy $E_{Z}$ can be extracted from the graph as indicated by the red arrow. The angles in this schematic diagram are exaggerated relative to $\mathbf{d}$. $\mathbf{f}$, Energy level schematic diagrams at the points labelled by the purple diamond and green star in $\mathbf{d}$ and $\mathbf{e}$.

The higher unloading rate is a consequence of the relatively small tunnel barrier between the two dots, as confirmed by numerical modelling. Our bound on the singlet loading rate places a weak bound on triplet-singlet relaxation of $\Gamma_{\mathrm{TS}}<63,000 \mathrm{~s}^{-1}$, although the actual value is expected to be much smaller ${ }^{16}$.

The phenomena described above of spin blockade and its complementary effect of LET can be unified by measurements of the system with an applied inplane magnetic field. In a magnetic field, the spin triplets are split linearly by the Zeeman energy $\left(E_{Z}=g \mu_{\mathrm{B}} B S_{Z}\right)$, where $\mu_{\mathrm{B}}$ is the Bohr magneton, $B$ is the magnetic field and $S_{Z}$ is +1 for $|\uparrow \uparrow\rangle,-1$ for $|\downarrow \downarrow\rangle$ and 0 for $(|\uparrow \downarrow\rangle+|\downarrow \uparrow\rangle) / \sqrt{2}$. As the $g$-factor is positive for silicon, the $\mathrm{T}_{-}$ states shift lower in energy compared with the $\mathrm{T}_{0}$ states, providing a technique for testing the interpretation of the data proposed above.

Figure $4 \mathrm{a}-\mathrm{c}(\mathrm{d}-\mathrm{f})$ shows the energy diagrams for the cases where $E_{Z}$ is less (more) than $E_{\mathrm{ST}}$. In Fig. $4 \mathrm{a}-\mathrm{c}$, the ground state of the $(1,1)$ configuration is $\mathrm{T}_{-}(1,1)$, whereas that of the $(2,0)$ configuration is $\mathrm{S}(2,0)$. Spin blockade now occurs in a smaller region than at $B=0$, as indicated by dashed lines and the orange circle in Fig. 4a. Spin blockade is lifted in the conventional way when $T_{-}(2,0)$ is lowered below $T_{-}(1,1)$, corresponding to the triangular regions on the lower right in Fig. 4a,b. Spin blockade is also lifted when the $S(1,1)$ state can participate in transport (blue diamond). However, the lifting of the blockade in this case is due to LET, because this $S(1,1)$ state is an excited state of the $(1,1)$ configuration, giving rise to a singlet tail in Fig. $4 \mathrm{a}, \mathrm{b}$. This tail is a striking example of a generalization of LET: the singlet-triplet splitting is now inverted, and the LET is now due to long lifetimes in the singlet channel rather than the triplet channel. LET can be generalized to any situation where electron transport occurs through long-lived excited states, whereas lower energy states that would be metastably trapped are avoided.

When $E_{Z}>E_{\mathrm{ST}}$ (Fig. $4 \mathrm{~d}-\mathrm{f}$ ), the ground state configurations are $T_{-}(1,1)$ and $T_{-}(2,0)$, and there should be no spin blockade because ground-state transitions are allowed (purple diamond). Our measurements indeed show full triangles with no blockade. From the features visible inside the triangles (bright lines parallel to base), various excited states can be identified. LET also occurs through excited spin singlet states in this configuration, giving rise to a tail (green star). These data demonstrate the existence of longlived electron spin states even in the presence of a finite magnetic field, a requirement for various quantum operations.

Received 1 February 2008; accepted 8 May 2008; published 8 June 2008.

\section{References}

1. Ono, K., Austing, D. G., Tokura, Y. \& Tarucha, S. Current rectification by Pauli exclusion in a weakly coupled double quantum dot system. Science 297, 1313-1317 (2002).

2. Johnson, A. C., Petta, J. R., Marcus, C. M., Hanson, M. P. \& Gossard, A. C. Singlet-triplet spin blockade and charge sensing in a few-electron double quantum dot. Phys. Rev. B 72, 165308 (2005). 
3. Koppens, F. H. L. et al. Control and detection of singlet-triplet mixing in a random nuclear field. Science 309, 1346-1350 (2005)

4. Rokhinson, L. P., Guo, L. J., Chou, S. Y. \& Tsui, D. C. Spin transitions in a small Si quantum dot. Phys. Rev. B 63, 035321 (2001).

5. Huttel, A. K. et al. Spin blockade in ground-state resonance of a quantum dot. Europhys. Lett. 62, 712-718 (2003).

6. Liu, H. W. et al. Pauli-spin-blockade transport through a silicon double quantum dot. Phys. Rev. B 77, 073310 (2008).

7. Petta, J. R. et al. Coherent manipulation of coupled electron spins in semiconductor quantum dots Science 309, 2180-2184 (2005)

8. Koppens, F. H. L. et al. Driven coherent oscillations of a single electron spin in a quantum dot. Nature 442, 766-771 (2006).

9. Kouwenhoven, L. P. et al. Excitation spectra of circular, few-electron quantum dots. Science 278, 1788-1792 (1997).

10. Kouwenhoven, L. P. et al. Mesoscopic Electron Transport (Kluwer, Dordrecht, 1997).

11. Ciorga, M. et al. Addition spectrum of a lateral dot from Coulomb and spin-blockade spectroscopy. Phys. Rev. B 61, 16315-16318 (2000).

12. Loss, D. \& DiVincenzo, D. P. Quantum computation with quantum dots. Phys. Rev. A 57, 120 (1998).

13. Vrijen, R. et al. Electron-spin-resonance transistors for quantum computing in silicon-germanium heterostructures. Phys. Rev. A 62, 012306 (2000).

14. Friesen, M. et al. Practical design and simulation of silicon-based quantum-dot qubits. Phys. Rev. B 67, 121301 (2003).

15. Elzerman, J. M. et al. Single-shot read-out of an individual electron spin in a quantum dot. Nature 430, 431-435 (2004)

16. Tahan, C., Friesen, M. \& Joynt, R. Decoherence of electron spin qubits in Si-based quantum computers. Phys. Rev. B 66, 035314 (2002).

17. de Sousa, R. \& Das Sarma, S. Theory of nuclear-induced spectral diffusion: Spin decoherence of phosphorus donors in Si and GaAs quantum dots. Phys. Rev. B 68, 115322 (2003).

18. Klein, L. J. et al. Coulomb blockade in a silicon/silicon-germanium two-dimensional electron gas quantum dot. Appl. Phys. Lett. 84, 4047-4049 (2004).

19. Sakr, M. R., Jiang, H. W., Yablonovitch, E. \& Croke, E. T. Fabrication and characterization of electrostatic Si/SiGe quantum dots with an integrated read-out channel. Appl. Phys. Lett. 87, 223104 (2005).

20. Gandolfo, D. S., Williams, D. A. \& Qin, H. Characterization of a silicon-germanium quantum dot structure at $4.2 \mathrm{~K}$ and $40 \mathrm{mK}$. J. Appl. Phys. 97, 063710 (2005).

21. Slinker, K. A. et al. Quantum dots in Si/SiGe 2DEGs with Schottky top-gated leads. New J. Phys. 7 , 246 (2005).
22. Berer, T. et al. Lateral quantum dots in Si/SiGe realized by a Schottky split-gate technique. Appl. Phys. Lett. 88, 162112 (2006)

23. Klein, L. J., Savage, D. E. \& Eriksson, M. A. Coulomb blockade and Kondo effect in a few-electron silicon/silicon-germanium quantum dot. Appl. Phys. Lett. 90, 033103 (2007).

24. Huttel, A. K., Ludwig, S., Eberl, K. \& Kotthaus, J. P. Spectroscopy of molecular states in a few-electron double quantum dot. Physica E 35, 278-284 (2006).

25. Gaudreau, L. et al. Stability diagram of a few-electron triple dot. Phys. Rev. Lett. 97, 036807 (2006).

26. Livermore, C., Crouch, C. H., Westervelt, R. M., Campman, K. L. \& Gossard, A. C. The Coulomb blockade in coupled quantum dots. Science 274, 1332-1335 (1996).

27. Blick, R. H. et al. Single-electron tunneling through a double quantum dot: The artificial molecule. Phys. Rev. B 53, 7899-7902 (1996).

28. van der Wiel, W.G. et al. Electron transport through double quantum dots. Rev. Mod. Phys. 75, 1 (2003).

29. Stopa, M. Quantum dot self-consistent electronic structure and the Coulomb blockade. Phys. Rev. B 54, 13767 (1996).

30. Hanson, R. et al. Single-shot readout of electron spin states in a quantum dot using spin-dependent tunnel rates. Phys. Rev. Lett. 94, 196802 (2005).

31. MacLean, K. et al. Energy-dependent tunneling in a quantum dot. Phys. Rev. Lett. 98, 036802 (2007).

Supplementary Information accompanies this paper on www.nature.com/naturephysics.

Acknowledgements

We thank Nick Leaf for help with the data acquisition programs. Support for this work was provided by NSA, LPS and ARO under contract number W911NF-04-1-0389, by the National Science

Foundation through DMR-0325634 and DMR-0520527, and by DOE through DE-FG02-03ER46028

Author contributions

Experimental work carried out by N.S., C.B.S., M.T., L.J.K., H.Q., H.L., D.E.S. and M.A.E. Data analysis carried out by N.S., C.B.S., A.J.R., R.J., M.F., R.H.B., S.N.C. and M.A.E. Project planning carried out by N.S., M.A.E., M.G.L., R.H.B., S.N.C., M.F., R.J. and D.E.S.

\section{Author information}

Reprints and permission information is available online at http://npg.nature.com/reprintsandpermissions Correspondence and requests for materials should be addressed to M.A.E. 